\title{
Mismatched Unrelated Donor
}

National Cancer Institute

\section{Source}

National Cancer Institute. Mismatched Unrelated Donor. NCI Thesaurus. Code C131678.

A donor who is not related to the transplant recipient, and who is not a human leukocyte antigen (HLA) tissue type match at one or more of the following loci: HLA-A, HLA-B, HLA-C, and HLA-DRB1. 